\title{
Designing and Validating a Questionnaire on Oral Health Care Knowledge, Attitude, and Practice of Dental Staff
}

\author{
Hediyeh Toutouni $i^{1 *} \mathbb{D}$, Seyed Ahmad Banihashem $\operatorname{Rad}^{2} \mathbb{D}$, Farzaneh Boroumand ${ }^{3,4} \mathbb{C}^{\mathbb{E}}$, Fatemeh Hosseini Pouya
}

'Department of Community Oral Health, School of Dentistry, Mashhad University of Medical Sciences, Mashhad, Iran.

2Department of Restorative, Preventive and Pediatric Dentistry, School of Dental Medicine, University of Bern, Switzerland.

${ }^{3}$ Department of Biostatistics, School of Health, Mashhad University of Medical Sciences, Mashhad, Iran.

${ }^{4}$ Department of Mathematics and Statistics, Faculty of Science and Engineering, Macquarie University, Sydney, Australia.

SPrivate Practice Dentist, Mashhad, Iran.

*Correspondence to: Hediyeh Toutouni (E-mail: toutounih@mums.ac.ir)

(Submitted: 04 August 2021 - Revised version received: 29 August 2021 - Accepted: 14 September 2021 - Published online: 26 October 2021)

\begin{abstract}
Objectives: This study aimed to design a valid questionnaire to evaluate knowledge, attitude, and practice (KAP) of Mashhad Dental school staff about oral health care and to assess the validity and reliability of this instrument.

Methods: The "Dental Staff Awareness of Oral Health Care" (DSAOHC) was divided into three concepts; knowledge, attitude and practice. Ten experts of Mashhad Dental School contributed to design the questionnaire and assessed the face and content validity (I-CVI and S-CVI/ AV) in two rounds. The necessity of each item was evaluated according to Lawshe's Content Validity Ratio (CVR). Construct validity, internal consistency, and reliability were assessed using confirmatory factor analysis, homogeneity coefficients, and test-retest by distributing the instrument among 207 dental staff working in other dental schools and private clinics.

Results: Based on the acceptable results of I-CVI, S-CVI/AV (>0.7), and CVR ( $>0.62)$, the final version of DSAOHC was approved with 34 questions, including 10 questions about background information, 8 questions for the knowledge, 5 questions for attitude and 11 questions related to practice. Cronbach's alpha coefficients were acceptable for all domains. The RMSEA criterion was $\leq 0.05$ and its upper band confidence interval (Cl 90\%) was $\leq 0.1$. Also, CFI indicated the suitability of the model (the desired value of CFI>0.9). Inter-Class Correlation/ ICC revealed the appropriate measure $($ ICC $=0.998)$.

Conclusion: The measures showed that this instrument is valid and reliable, also culturally adjusted to and acceptable for this community. It may adequately evaluate staffs' knowledge, attitude, and practice toward oral health.

Keywords: Oral health, knowledge, attitude, practice, questionnaire
\end{abstract}

\section{Introduction}

Employees are the human resources of organizations, including the health system. The staff's awareness of health issues affects directly their own well-being and indirectly satisfaction of their clients. ${ }^{1,2}$ Dental caries and periodontal diseases have been concerned as the most important oral health burdens among all ages in Iran like other countries. ${ }^{3-5}$ These two diseases are the main causes of tooth loss as their treatment is costly. ${ }^{6}$ Clients satisfaction toward a system directly depends on the performance of the system's staff in terms of accountability, proper communication with patients (empathy), and staffs' readiness to manage the challenges in the system. Providing facilities, understanding the necessity of treatment and its process along with urgency, ability to be more empathetic, and giving clear and simple explanations to patients are critical for treatment process which would certainly affect patients' satisfaction as a part of chair side manner. ${ }^{2,7}$ Several factors affect the relationship between staff and patients containing staff's awareness of related health issues and health promotion programmes. ${ }^{2}$

On the other hand, the proper planning for maintaining and improving the staff's oral health, their knowledge, attitude, and practice which are also needed for appropriate dental reform in a dental setting, requires a survey at first place, which measures their awareness towards oral health care. At further stages, an assessment of their oral health status and their treatment needs in terms of dental caries and periodontal diseases are needed. Oral health care is divided into two dimensions which are related to hard tissue and soft tissue. Dental caries is the most common multifactorial infectious oral disease and the leading cause of tooth decay/tooth loss. ${ }^{8}$ Because of the multifactorial aetiology, there are different approaches to prevent this disease. ${ }^{9}$ Periodontal diseases are the most common oral illness that influence soft tissues such as gingiva and periodontium. Not only the localized/oral circumstances can result in periodontal diseases, but also there are systemic diseases that lead to inflammatory conditions in periodontium. ${ }^{10,11}$

There are several KAP instruments to measure the knowledge, attitude, and practice of different groups of people about oral health. The target groups of these questionnaires are school children, health care workers, mothers, and people who are suffering from systemic diseases. ${ }^{12-19}$ Since, the contents of those questionnaires were general and not adequate for oral health care workers; also, they primarily focused on the most common oral hygiene habit such as tooth brushing, and few questions were related to diet as an important risk factor of dental caries, this study aimed to design and validate a questionnaire to measure knowledge, attitude, and practice of the Dental School staff who graduated from Nursing School and similar disciplines, but work as oral health care providers.

This study was the first phase of a health promotion programme for Mashhad Dental School staff and the validated instrument will use in the further surveys, annually.

\section{Materials and Methods}

Before developing an instrument, six databases (Pubmed, Embase, BioMedCentral, and Google scholar for English 
resources and Scientific Information Database (SID) for Persian articles) were searched to find a valid and reliable pre-existing questionnaire to measure knowledge, attitude, and practice (KAP) of oral health care staff at dental settings. The search was limited to English and Persian literature published between 1990 and 2021. Keywords used for conducting this search were "healthcare", "design", "validation", "questionnaire", "knowledge", "attitude", "practice", and "oral health". Based on a search conducted 239, 155, 445, and 23700 articles were obtained from the first five abovementioned databases, respectively. There was not found any Persian article. Among the papers found, irrelevant studies were omitted considering their titles, duplicate articles were removed, and only studies, which were conducted on developing and designing the valid questionnaires, were remained. There was no valid preexisting questionnaire for the purpose of this study.

\section{Face and Content Validity}

The "Dental Staff Awareness of Oral Health Care" (DSAOHC) was divided into three concepts; knowledge, attitude, and practice. To determine the indicators of each concept, we checked out the adults' KAP questionnaires, oral health-related textbooks, dental public health articles, and also resources from the American Dental Assistants Association. ${ }^{20-22}$ Then, this initial draft was sent to the professors of Dental Public Health and Periodontics to add items that they considered necessary.

After modifying the first draft, ten experts of Mashhad Dental School have also assessed the face and content validity of the questionnaire. In addition, ten dental workforce checked questions in terms of face validity as a target group, and two questions were revised according to their comments. These ten people did not take part in the following phases of the study.

The criteria for selecting experts was contained: their background in writing and translation in scientific publications, research and teaching experiences, familiarity with the educational needs of the target population, and also with the developing process of a valid instrument as a questionnaire. ${ }^{23,24}$

The content validity of the questionnaire was evaluated by experts using the Content Validity Index (I-CVI and S-CVI/ $\mathrm{AV})^{25}$ and the necessity of each item was scored with Lawshe's Content Validity Ratio (CVR). ${ }^{26}$ Content Validity Index was calculated for each question. Experts rated each item on a 4-point scale from 1 to 4 that: score 1 was the lowest and score 4 represented the highest of each measurement item (relevancy to the subject, the simplicity, and clarity of each item). This four-point scale was also used to avoid shifting to the midpoint. ${ }^{25}$ The questions that obtained very low CVR were omitted based on experts' opinions. The items which met moderate levels for CVR or zero (means that half of the experts indicated those items as "essential"), but they showed high relevancy, clarity, and simplicity remained in the questionnaire and were modified and sent for the experts after revision. I-CVI, S-CVI/Ave, and CVR were calculated after the second review.

The content validity of the questionnaire and the I-CVI score for each indicator were calculated using the following formula. For example, the score of relevancies for each indicator was calculated which included (score 4) a highly relevant score, and those that are relevant but in need of review gets (score 3) divided by the total number of experts. The same method was used to calculate the I-CVI of each indicator in terms of utility (clarity and simplicity). Each indicator with an I-CVI score above 0.78 for each of the three domains of relevancy to subject, clarity, and simplicity was retained in the questionnaire. This score of 0.78 was determined by the contribution of 10 experts by considering valid scientific papers. ${ }^{25}$

$$
\mathrm{I}-\mathrm{CVI}=\frac{\text { score }\left(\begin{array}{c}
\text { related but in need of review } \\
\text { +highly related }
\end{array}\right)}{\text { number of experts }}
$$

After calculating I-CVI for all indicators (questions), the content validity of the whole questionnaire was determined using S-CVI/Ave index and calculating the mean of each I-CVI value. ${ }^{25}$

Content validity ratio (CVR) was calculated according to the number of experts and also those who had selected the "essential" option for each indicator.

$$
\mathrm{CVR}=\left(\mathrm{N}_{\mathrm{e}}-\mathrm{N} / 2\right) /(\mathrm{N} / 2)
$$

$N_{e}$ is the number of panel members indicating an item "essential," and $N$ is the number of panel members. According to the number of experts $(n=10)$, the minimum content validity ratio which would be accepted was $0.62 .{ }^{26}$

After confirming the face and content validity by the experts, questionnaires were distributed among 207 dental clinic staff working in Mashhad, Bojnord, and Birjand Dental Schools in a similar mean age group to verify the construct validity, to conduct factor analysis, and to assess the over-scale and over-time reliability.

\section{Homogeneity, Construct Validity, and Test-retest Reliability}

Questionnaires were filled out by 207 dental staff. The homogeneity (internal consistency) of the questionnaire was determined by calculating $\alpha$ Cronbach for Likert scale, and Kuder Richardson-20 (KR-20) for dichotomous items (Knowledge) because of their different nature, in which there were true answers among choices. ${ }^{27}$ The acceptable cut-off considered for $\alpha$ Cronbach was $0.7 .{ }^{28}$ Over-time reliability of the instrument was assessed by Test-Retest after filling out the redistributed questionnaires by 50 out of 207 people of the same population in a two-week interval.

We applied a Confirmatory Factor Analysis (CFA) to investigate construct validity. Tow goodness of fit criteria including comparative fit index (CFI) and root-mean-squared error of approximation (RMSEA) was reported. The CFI is an index between 0 and 1. A greater value indicates a better fit. The RMSEA shows the simplicity of the models. The closer the value to 0 shows the model has a better fit. A good model has a value of 0.08 or lower. We carried out the CFA in Amos 24 software.

Figure 1 shows the process of questionnaire development.

\section{Ethical Considerations}

This study was approved by the Ethical Committee of Mashhad dental school at Mashhad University of Medical Sciences (No. IR.mums.sd.REC.1394.3272971). All the employees were informed about the study and were invited to complete the questionnaire. No one was forced to participate in the study. Moreover, all questionnaires were coded, and data were entered into the software according to their codes were documented on the questionnaires. All data are being kept confidential. 


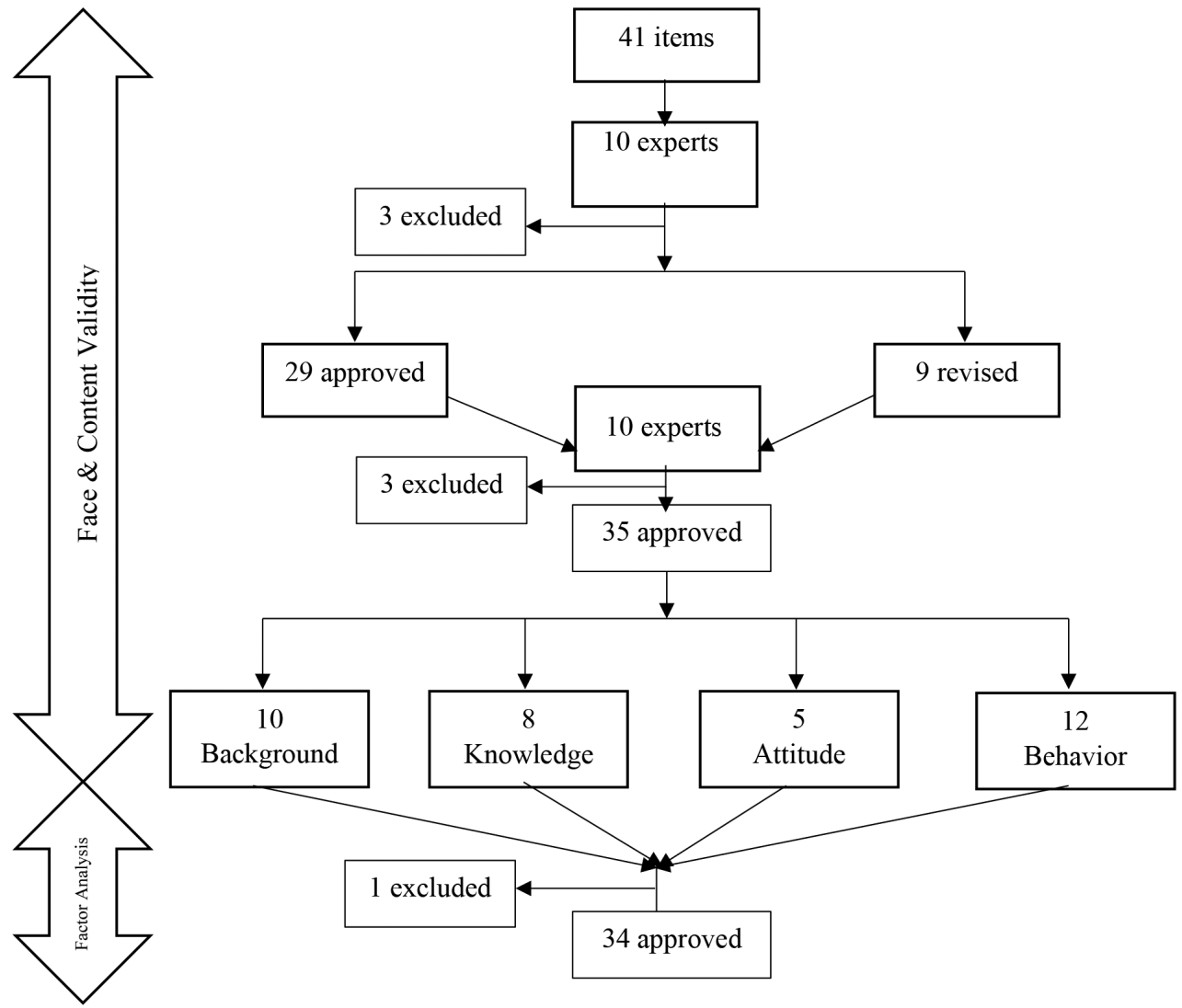

Fig. 1 The flowchart of two phases of the study. Phase I: I-CVI, S-CVI and CVR calculation by 10 experts. Phase II: Factor analysis and reliability tests among 207 participants.

\section{Results}

\section{Face and Content Validity}

The preliminary assessment of the Content Validity Ratio and Construct Validity by 10 experts showed the need for making changes in the questionnaire. Eleven out of 41 questions were asked about background information. Among 30 the remaining questions, eight indicators were related to knowledge, six to attitude, and 16 indicators went to practice. One indicator of background section and two indicators of practice were excluded from the questionnaire based on experts opinions and due to low CVIs and CVRs. Twenty-nine indicators were approved with appropriate CVI and CVR scores and nine indicators were rephrased due to the high CVI score, low CVR (Table 1).

After reviewing for the second time by the experts, the questionnaire was approved with 35 questions, including 10 questions about background information, eight questions related to the concept of knowledge, five questions for the concept of attitude and 12 questions for the concept of practice. The amounts of I-CVI, S-CVI/AV, and CVR for the instrument were $\geq 0.79$ for each item, 0.85 , and $\geq 0.62$, respectively (Table 2 ).

\section{Internal Consistency, Construct Validity, and Test-retest Reliability}

The mean age of 207 participants, for conducting the factor analysis, was $52.9( \pm 26.6)$. They consisted of 146 (71\%) women and $61(29 \%)$ men whose average years of experience in a dental setting were $6.6 \pm 7.07$ in which $97(46.9 \%)$ already took part in an educational course on oral health care. Out of 207 participants, $100(48.3 \%)$ worked in clinical, $82(39.6 \%)$ in administrative settings and $18(8.7 \%)$ worked in services (seven participants did not reply to this question). In terms of the level of education, 111(53.6\%) had Master and 34(16.4\%) Bachelor degrees in health sciences. In addition, 56(27.1\%) had a Diploma degree and the level of education of 6(3\%) was under Diploma.

The RMSEA criterion was less than 0.05 and its confidence interval (CI 90\%) was less than 0.1. Another criterion, CFI (Comparative Fit Index), also indicated the suitability of the model (the desired value of CFI $>0.9$ ).

The statistical analysis showed that if the question PQ5 was removed, the Cronbach $\alpha$ value of the scale would increase from 0.62 to 0.661 . However, Kuder Richardson-20 (KR-20) analysis showed low homogeneity between eight questions related to the concept of knowledge (KR-20 $=0.25)$.

Internal consistency was recalculated after PQ5 was deleted and showed that was acceptable (close to 0.7). As a result, the question PQ5 was removed from the final instrument. The overtime reliability of the questionnaire was confirmed by ICC = 0.985 (CI 90\% $=0.972-0.992)$ (Table 3$)$.

\section{Discussion}

The Dental Staff Awareness of Oral Health Care (DSAOHC) questionnaire which was developed in this study showed acceptable measures as a standard instrument to evaluate the knowledge, attitude, and practice of the oral health care workforce. I-CVI and S-CVI/AV in all 34 remained items of the questionnaire met acceptable cut-offs [Polit-Lawshe-Grant]. Furthermore, Cronbach $\alpha$ and ICC presented acceptable ${ }^{27}$ and excellent values. ${ }^{29}$ 


\begin{tabular}{|c|c|c|c|c|c|c|}
\hline No. & Item question & Relevancy & Clarity & Simplicity & CVR & Interpretation \\
\hline KQ1 & The number of permanent teeth in human & 0.9 & 0.8 & 0.9 & 0.4 & Rephrased \\
\hline KQ2 & Which food product can cause dental caries & 1 & 1 & 0.8 & 0.8 & Included \\
\hline KQ3 & Which one is correct about dental calculus & 0.9 & 0.8 & 0.8 & 0.8 & Included \\
\hline KQ4 & When should we go to the dentist & 1 & 0.9 & 0.8 & 0.8 & Included \\
\hline KQ5 & Which one is the most important time for toothbrushing & 0.9 & 0.8 & 0.7 & 0.8 & Included \\
\hline KQ6 & Which one is the common cause of malodor & 1 & 0.9 & 0.8 & 1 & Included \\
\hline KQ7 & What are the causes of dental caries (please order from the most to the least) & 1 & 0.8 & 0.8 & 1 & Included \\
\hline KQ8 & $\begin{array}{l}\text { What are the prevention methods for dental caries (please order from the } \\
\text { most to the least) }\end{array}$ & 0.9 & 0.7 & 0.7 & 0.8 & Included \\
\hline AQ1 & I believe in good oral health may lead to more social popularity & 0.8 & 0.8 & 0.7 & 0.4 & Rephrased \\
\hline AQ2 & I believe in toothbrushing can reduce periodontal diseases & 1 & 1 & 0.9 & 0.8 & Included \\
\hline AQ3 & I believe that toothbrushing eliminates malodor & 1 & 0.9 & 0.9 & 0.8 & Included \\
\hline AQ4 & I believe that oral health care can prevent some gastrointestinal diseases & 0.7 & 0.8 & 0.7 & 0.0 & Rephrased \\
\hline AQ5 & I believe that flossing is effective for preventing dental caries & 1 & 1 & 1 & 0.8 & Included \\
\hline AQ6 & I believe that pregnancy can be the reason for tooth loss & 0.9 & 0.8 & 0.8 & 0.8 & Included \\
\hline PQ1 & How often do you brush your teeth & 1 & 0.8 & 0.9 & 0.8 & Included \\
\hline PQ2 & How often do you floss your teeth & 0.9 & 0.9 & 0.9 & 0.8 & Included \\
\hline PQ3 & How often do you use a fluoride mouthwash & 0.8 & 0.9 & 0.9 & 0.4 & Rephrased \\
\hline PQ4 & Do you brush all your teeth surfaces & 0.9 & 0.7 & 0.9 & 0.6 & Included \\
\hline PQ5 & Do you break shelled nuts using your teeth & 0.8 & 0.9 & 0.9 & 0.4 & Rephrased \\
\hline PQ6 & Do you have a dental appointment each year regularly & 1 & 1 & 1 & 0.8 & Included \\
\hline PQ7 & Do you clean your tongue after toothbrushing & 0.8 & 0.9 & 0.9 & 0.4 & Rephrased \\
\hline PQ8 & Do you brush your teeth at the workplace after lunch & 0.9 & 0.9 & 0.9 & 0.4 & Rephrased \\
\hline PQ9 & Do you brush your teeth if there is no toothpaste & 0.5 & 0.8 & 0.8 & -0.2 & Deleted \\
\hline PQ10 & Do you rinse your entire mouth after toothbrushing & 0.6 & 0.8 & 0.8 & 0.0 & Rephrased \\
\hline PQ11 & Do you eat or drink something after toothbrushing at night & 1 & 1 & 1 & 0.6 & Included \\
\hline PQ12 & How much toothpaste do you use for brushing your teeth each time & 0.6 & 0.75 & 0.78 & 0.4 & Rephrased \\
\hline PQ13 & Which kind of toothbrush do you usually use & 0.8 & 0.8 & 0.8 & 0.6 & Included \\
\hline PQ14 & Where do you keep your toothbrush & 0.7 & 0.6 & 0.8 & -0.2 & Deleted \\
\hline PQ15 & How often do you change your toothbrush & 0.9 & 1 & 0.9 & 0.8 & Included \\
\hline PQ16 & Why do you floss your teeth & 0.9 & 0.8 & 0.9 & 0.8 & Included \\
\hline
\end{tabular}

\begin{tabular}{llccccc}
\hline \multicolumn{2}{l}{ Table 2 . } & Assessment of I-CVI, $\mathbf{S}$-CVI, and CVR by expert panel (2 ${ }^{\text {nd }}$ time) & & & \\
\hline No. & Item question & Relevancy & Clarity & Simplicity & CVR & Decision \\
\hline KQ1 & The number of permanent teeth in human & 0.9 & 0.8 & 1 & 0.6 & Included \\
AQ1 & I believe in good oral health may lead in more social popularity & 0.9 & 0.9 & 0.8 & 0.8 & Included \\
AQ4 & I believe that tooth loss can lead in some gastrointestinal diseases & 0.7 & 1 & 0.7 & 0.0 & Deleted after Panel \\
PQ3 & How often you use mouthwash & 0.9 & 1 & 0.9 & 0.6 & Included \\
PQ5 & Do you break shelled nuts using your teeth & 1 & 1 & 0.9 & 0.8 & Included \\
PQ7 & Do you clean your tongue after toothbrushing & 0.8 & 1 & 0.9 & 0.6 & Included \\
PQ8 & Do you brush your teeth at workplace after lunch & 1 & 1 & 0.9 & 0.8 & Included \\
PQ10 & Do you rinse your entire mouth after toothbrushing & 0.9 & 0.9 & 0.8 & 0.2 & Deleted \\
PQ12 & How much toothpaste do you use for brushing your teeth each time & 0.9 & 0.75 & 0.78 & 0.2 & Deleted \\
\hline
\end{tabular}




\begin{tabular}{|c|c|c|c|c|c|c|c|}
\hline Section & & ICC & Lower bound Cl 90\% & Upper bound Cl 90\% & True value & $\mathbf{d f}_{1}$ & $\mathrm{df}_{2}$ \\
\hline \multirow[t]{2}{*}{ Background } & Item & 0.989 & 0.980 & 0.994 & 181.210 & 42 & 42 \\
\hline & Average & 0.995 & 0.990 & 0.997 & 181.210 & 42 & 42 \\
\hline \multirow[t]{2}{*}{ Knowledge } & Item & 0.991 & 0.983 & 0.995 & 222.175 & 49 & 49 \\
\hline & Average & 0.995 & 0.991 & 0.997 & 222.175 & 49 & 49 \\
\hline \multirow[t]{2}{*}{ Attitude } & Item & 0.708 & 0.536 & 0.823 & 5.757 & 49 & 49 \\
\hline & Average & 0.829 & 0.698 & 0.903 & 5.757 & 49 & 49 \\
\hline \multirow[t]{2}{*}{ Behavior } & Item & 0.895 & 0.822 & 0.939 & 17.992 & 49 & 49 \\
\hline & Average & 0.944 & 0.902 & 0.968 & 17.992 & 49 & 49 \\
\hline \multirow[t]{2}{*}{ Total } & Item & 0.970 & 0.945 & 0.984 & 66.373 & 49 & 49 \\
\hline & Average & 0.985 & 0.972 & 0.992 & 66.373 & 49 & 49 \\
\hline
\end{tabular}

Having revised the first draft of the instrument, five questions were added to demographic data (gender, age, date of birth, level of education) as background information. These questions asked about "years of experience", "work field" (clinical, official or services), "participating in an educational course/workshop on oral health care", "systemic diseases", and "tobacco usage". As "years of experience", "working in an official or clinical department", and "participation in an oral health education course" will make changes in the interpretation of the results and decision making for the following oral health promotion programmes. The other two questions, which more focused on medical history and oral habits of the staff, were considered for detailed comparison and valid interpretation in the further clinical phases of the study.

In the second part of the questionnaire (concept of knowledge), eight questions were designed from general knowledge about dental caries and periodontal diseases to their causation and prevention. In previous studies, these questions were mostly based on general knowledge of the population ("brushing habits" and "frequency of dental appointments") and their type was multiple choice. ${ }^{15,16}$ The first six questions in the concept of knowledge, were designed as multiple choice questions, but two further questions were developed so that respondents are supposed to prioritize in order the aforementioned causes of dental caries and its prevention methods, from the most likely to the least (1 to 4 ). The multifactorial nature of dental caries was the rationale to ask the questions in this form. ${ }^{30}$ Also, the attributable risk of each factor was previously determined in studies ${ }^{30,31}$ and oral health care staff should know the hierarchy of them. ${ }^{20}$ The low homogeneity coefficient (KR-20) between the questions which were designed to measure the knowledge confirmed the multifactorial nature of dental caries and multidimensional character of oral health. Each of eight questions referred to one of the aspects of oral health. The questions about "the number of permanent teeth" was a piece of general knowledge about the normal oral status and other questions related to the different subjects such as diet and snacking, regular toothbrushing, dental hygiene and calculus, malodor, appropriate dental visit interval, the causes and prevention methods of dental caries. This variety among questions led to low homogeneity (low KR-20 coefficient), which means that it cannot be predicted that if respondents chose the correct answer for the first three questions, they would choose the true answer for the following questions as well because these questions measure different aspects of oral health care knowledge. Moreover, when the number of items on the scale is less than 10 , it may cause homogeneity to be low. ${ }^{27}$ However, increasing the number of items could lead to a decrease in the number of respondents. ${ }^{27}$ Given that the aim, each item would be assessed separately. Regarding our research questions and given that all eight items reflect the basics of theoretical aspects of oral health care, items were retained despite their poor homogeneity coefficient. ${ }^{28}$

Five out of six questions which were developed in the first draft to assess the attitude remained in the final instrument in the form of 4-scale Likert (from strongly agree to strongly disagree). These questions ask the respondents' attitudes about "oral hygiene and social popularity", "brushing and periodontal diseases", "brushing and halitosis", "flossing and dental caries", and "pregnancy and tooth decay". A four-scale type of Likert was preferred in this section to avoid the inclination of responses to the midpoint (neutral responses). Question AQ4 was deleted in the second revision.

Given that the Cronbach $\alpha$ coefficient improved after removing one question (PQ5), 11 questions remained to assess practice. Question PQ5 was "Do you use your teeth to crack hard-shelled nuts such as hazelnut, walnut, pistachio?" and after its deletion, the items of the instrument were more closely related ( 0.62 vs 0.661$)$. The left items asked about "brushing all teeth surfaces", "annual dental appointments", "cleaning the tongue", "brushing after lunch in the workplace", "eating or drinking after brushing at nights", "type of their toothbrush bristles (soft/medium/hard)", "their primary goal of flossing", and "changing toothbrush frequency".

In comparison with a similar study performed for validating a KAP instrument to measure it among health care professionals, the results of this study show high content validity indices as well and lower internal consistency. Wong modified and validated a questionnaire to measure the KAP of healthcare providers in elderly residences. ${ }^{32}$ This questionnaire was translated and retranslated from English into Chinese and vice versa and all amounts of I-CVI, S-CVI/Ave, S-CVI/UA, and CVR were in the excellent range $(1.00,1.00,1.00$, and $>0.99$, respectively). In addition, internal consistency showed good results for all three concepts $(\mathrm{K}=0.67, \mathrm{~A}=0.93$, and $\mathrm{P}=0.92)$. The questions more focused on tooth brushing and oral hygiene among old residents, denture care, and also dental 
visit in all domains. The high internal consistency of the instrument may be related to the point that this instrument already passed validation processes and was frequently utilized previously. The Chinese version tested its valid feasibility in a new context. On the other hand, all items were mostly confined to one subject which was oral hygiene (brushing teeth and cleaning dentures) of elderly people.

\section{Conclusion}

The DSAOHC questionnaire can be a useful instrument to assess staff awareness at the beginning of the recruitment at dental school and help them to find the necessary courses and oral health care training to improve their self-care, which would indirectly affect their responsiveness and patient satisfaction. The data from this questionnaire will assist educational supervisors and managers to establish oral health courses.

\section{Acknowledgment}

This study was ethically approved by Ethical Committee at Mashhad University of Medical Sciences under the following code: IR.mums.sd.REC.1394.327, and was financially supported by the Research Deputy of Mashhad Dental School under research number: 960596 . The authors would like to express their gratitude to the Educational Supervisor and Director of Nursing Services, Mrs Jalalian and Mr Khorashadi, for their support during this study. Also, the sincere thanks of the authors go to the faculty members of Mashhad Dental School who spent time reading and assessing the instrument.

\section{Conflicts of Interest}

Authors have no conflict of interest.

\section{References}

1. Lombardi DN, Keramer B, Schermerhorn JR. Health care management. The United States of America: Wiley; 2007.

2. Roberts $M$, Hsiao W, Berman P, Reich M. Getting health reform right: a guide to improving performance and equity. $1^{\text {st }}$ ed. Oxford: Oxford university press;2003.

3. Jalaleddin $\mathrm{H}$, Ramezani GH. Prevalence of gingivitis among school attendees in Qazvin, Iran. East Afr J public health. 2009:6(2):171-4.

4. Phantumvanit P, Makino Y, Ogawa H, Rugg-Gunn A, Moynihan P, Petersen P.E, Evans W, Feldens CA, Lo E, Khoshnevisan M.H, Baez R, Varenne B, Vichayanrat T, Songpaisan Y, Woodward M, Nakornchai S, Ungchusak C. WHO global consultation on public health intervention against early childhood caries. Community Dent Oral Epidemiol. 2017;13:280-288.

5. Peres MA, Macpherson LMD, Weyant RJ, Daly B, Venturelli R, Mathur MR, Listl S, Celeste RK, Guarnizo-Herreno CC, Kearns C, Benzian H, Allison P, Watt RG. Oral diseases: a global public health challenge. Lancet. 2019;394:249-60.

6. Phipps KR, Stevens VJ. Relative contribution of caries and periodontal disease in adult tooth loss for an HMO dental population. J Public Health Dent. 1995:55(4):250-252.

7. Guo KL. DECIDE: A decision-making model for more effective decision making by health care managers. Health Care Manag. 2020. 39(3):133-141.

8. Selwitz RH, Ismail Al, Pitts N. Dental caries. Lancet. 2007;369:51-9.

9. Yadav KH, Prakash S. Dental caries: A review. Asian J Biomed Pharmaceut Sci. 2016: 6(53):1-7.

10. Hajishengallis $G$, Chavakis T. Local and systemic mechanisms linking periodontal disease and inflammatory comorbidities. Nat Rev Immunol. 2021;21:426-440.

11. Kinane D, Stathopoulou P, Papapanou P. Periodontal diseases. Nat Rev Dis Primers. 2017;3:1-14.

12. Al-Omiri MK, Al-Wahadni AM, Saeed KN. Oral health attitudes, knowledge, and behavior among school children in North Jordan. J Dent Educ 2006;70(2):179-187.

13. Rad M, Shahravan A, Haghdoust A. Designing a valid questionnaire on oral health knowledge, attitude, and practice in 12 year-old children in Iran. Mazandaran Uni Med Sci. 2015;15(126):130-133.

14. Hajmohammadi A. Assessing knowledge, attitude, and behavior of health workers in public health care centers in Kerman, Iran. J KUMS 2010:50. (Persian)

15. Pourhashemi J. A survey on the knowledge of health system personnel in Ghom province toward oral and dental health. J Dent Med. 2004;17(3):77-82.

16. Kawamura M, Iwamoto Y. Present state of dental health Knowledge, attitudes, behaviour and perceived oral health of Japanese employees. Int Dent J. 1999:49(3):173-81.
17. Wardh I, Jonsson M, Wikstrom M. Attitudes to and knowledge about oral health care among nursing home personnel- an area in need of improvement. Gerodontology. 2012;29:787-792.

18. Nardi GM, Giraldi G, Lastella P, La Torre G, Saugo E, Ferri F, Pacifici L, Ottolenghi L, Guerra F, Polimeni A. Knowledge, attitudes and behavior of Italian mothers towards oral health: questionnaire validation and results of a pilot study. Annali di Stomatologia. 2012;ill (2):69-74.

19. Rasouli-Ghahroudi AA, Rokn AR, Khorsand A, Aghajani H, Amini A, Shamshiri AR, Rahimi H, Kabir A. Designing and standardizing a questionnaire for evaluating knowledge, attitude, and practice of Iranian adults with cardiovascular diseases about oral health. ARYA Atheroscler. 2013;9(6):350-356.

20. Phinney DJ, Halstead JH. Delmar's Dental Assisting: A comprehensive approach. United States:Thomson Delmar Learning; 2000.

21. American Dental Assistants Association. Continueuing education materials; 2019 [updated 2021]. Available from: https://www.adaausa.org/Education/ Continuing-Education/

22. Daly B, Batchelor P, Treasure E, Watt R. Essentials dental public health $2^{\text {nd }}$ edition. USA: Oxford University Press; 2013.

23. Garson GD. Validity and reliability. USA: Statistical Associates Publishers; 2013

24. Grant JS, Davis LL. Selection and use of content experts for instrument development. Res Nurs Health. 1997;20(3):269-274.

25. Polit DF, Beck CT. The content validity index: are you sure you know what's being reported? Critique and recommendations. Rese Nurs Health. 2006:29(5):489-497.

26. Lawshe $\mathrm{CH}$. A quantitative approach to content validity'. Personnel psychology. 1975:28(4):563-575.

27. Bolarinwa OA. Principles and methods of validity and reliability testing of questionnaires used in social and health science researches. Niger Postgrad Med J. 2015:22:195-201.

28. Rattray J, Jones MC. Essential elements of questionnaire design and development. J Clin Nurs. 2006:16:234-243.

29. Koo TK, Li YM. A guideline of selecting and reporting intraclass correlation coefficients for reliability research. J Chiropr Med. 2016:15:155-163.

30. Fejerskov O, Nyvad B, Kidd E. Dental caries: The disease and its clinical management. 3rd ed. Oxford: Willey Blackwell; 2015.

31. Attaran N, Khoshnevisan MH, Ghorbani Z, Pakkhesal M, Dehghanian D. Dental caries predictors in countries with different human development index: A review of articles. J Int Oral Health. 2016:8(2):182-190.

32. Wong FMF. First data in the process of validating a tool to evaluate Knowledge, Attitude, and Practice of healthcare providers in oral care of institutionalized elderly residents: content validity, reliability and pilot study. Int J Environ Res Public Health. 2021:18:1-15. 